\title{
Caffeine-stimulated fatty acid oxidation is blunted in CD36 null mice
}

Citation for published version (APA):

Lally, J. S. V., Jain, S. S., Han, X. X., Snook, L. A., Glatz, J. F. C., Luiken, J. J. F. P., McFarlan, J., Holloway, G. P., \& Bonen, A. (2012). Caffeine-stimulated fatty acid oxidation is blunted in CD36 null mice. Acta Physiologica, 205(1), 71-81. https://doi.org/10.1111/j.1748-1716.2012.02396.x

Document status and date:

Published: 01/05/2012

DOI:

10.1111/j.1748-1716.2012.02396.x

Document Version:

Publisher's PDF, also known as Version of record

Document license:

Taverne

Please check the document version of this publication:

- A submitted manuscript is the version of the article upon submission and before peer-review. There can be important differences between the submitted version and the official published version of record.

People interested in the research are advised to contact the author for the final version of the publication, or visit the DOI to the publisher's website.

- The final author version and the galley proof are versions of the publication after peer review.

- The final published version features the final layout of the paper including the volume, issue and page numbers.

Link to publication

\footnotetext{
General rights rights.

- You may freely distribute the URL identifying the publication in the public portal. please follow below link for the End User Agreement:

www.umlib.nl/taverne-license

Take down policy

If you believe that this document breaches copyright please contact us at:

repository@maastrichtuniversity.nl

providing details and we will investigate your claim.
}

Copyright and moral rights for the publications made accessible in the public portal are retained by the authors and/or other copyright owners and it is a condition of accessing publications that users recognise and abide by the legal requirements associated with these

- Users may download and print one copy of any publication from the public portal for the purpose of private study or research.

- You may not further distribute the material or use it for any profit-making activity or commercial gain

If the publication is distributed under the terms of Article $25 \mathrm{fa}$ of the Dutch Copyright Act, indicated by the "Taverne" license above, 


\title{
Caffeine-stimulated fatty acid oxidation is blunted in CD36 null mice
}

\author{
J. S. V. Lally,' $\quad$ S. S. Jain,' X. X. Han, ' L. A. Snook,' J. F. C. Glatz, ${ }^{2}$ J. J. F. P. Luiken, ${ }^{2}$ \\ J. McFarlan,' G. P. Holloway' and A. Bonen' \\ I Department of Human Health and Nutritional Science, University of Guelph, Guelph, ON, Canada \\ 2 Department of Molecular Genetics, Maastricht University, Maastricht, the Netherlands
}

Received 26 June 2011 , revision requested 7 September 2011 ,

revision received I November 2011,

accepted 5 December 2011

Correspondence: A. Bonen, PhD, Department of Human Health and Nutritional Sciences, University of Guelph, Guelph, ON, Canada NIG 2WI.

E-mail: arend.bonen@gmail.com

\begin{abstract}
Aim: The increase in skeletal muscle fatty acid metabolism during exercise has been associated with the release of calcium. We examined whether this increase in fatty acid oxidation was attributable to a calcium-induced translocation of the fatty acid transporter CD36 to the sarcolemma, thereby providing an enhanced influx of fatty acids to increase their oxidation.

Methods: Calcium release was triggered by caffeine $(3 \mathrm{~mm})$ to examine fatty acid oxidation in intact soleus muscles of WT and CD36-KO mice, while fatty acid transport and mitochondrial fatty acid oxidation were examined in giant vesicles and isolated mitochondria, respectively, from caffeine-perfused hindlimb muscles of WT and CD36-KO mice. Western blotting was used to examine calcium-induced signalling.

Results: In WT, caffeine stimulated muscle palmitate oxidation $(+136 \%)$, but this was blunted in CD36-KO mice $(-70 \%)$. Dantrolene inhibited (WT) or abolished (CD36-KO) caffeine-induced palmitate oxidation. In muscle, caffeine-stimulated palmitate oxidation was not attributable to altered mitochondrial palmitate oxidation. Instead, in WT, caffeine increased palmitate transport $(+55 \%)$ and the translocation of fatty acid transporters CD36, FABPpm, FATP1 and FATP4 (26-70\%) to the sarcolemma. In CD36-KO mice, caffeine-stimulated FABPpm, and FATP1 and 4 translocations were normal, but palmitate transport was blunted $(-70 \%)$, comparable to the reductions in muscle palmitate oxidation. Caffeine did not alter the calcium-/calmodulin-dependent protein kinase II phosphorylation but did increase the phosphorylation of AMPK and acetyl-CoA carboxylase comparably in WT and CD36-KO.
\end{abstract}

Conclusion: These studies indicate that sarcolemmal CD36-mediated fatty acid transport is a primary mediator of the calcium-induced increase in muscle fatty acid oxidation.

Keywords AMPK, calcium, CaMK, CD36, mitochondria, transport
Skeletal muscle fatty acid utilization is highly dependent on their uptake from the circulation, as intramuscular triacylglycerol depots provide only approx. $10 \%$ of the fatty acids destined for $\beta$-oxidation (Kiens 2006). CD36 is a well-studied plasma membrane fatty acid transporter that is important in lipid handling in a range of tissues, including skeletal muscle (Bonen et al. 1998, Glatz et al. 2010). CD36 has been found in intracellular depots from which it can be induced to translocate to the cell surface in 
response to metabolic perturbations, including exposure to insulin, 5-aminoimidazole-4-carboxamide-1- $\beta$ D-ribofuranoside (AICAR) and muscle contraction (Bonen et al. 2000, Luiken et al. 2002, Jain et al. 2009). Studies using CD36 null mice have established that the ablation of CD36 has a number of effects on whole body (Febbraio et al. 1999), cardiac (Habets et al. 2007) and skeletal muscle fatty acid metabolism (Bonen et al. 2007), such as a reduction in AICARand insulin-mediated plasmalemmal fatty acid transport and a decrease in AICAR-stimulated fatty acid oxidation (Bonen et al. 2007).

Calcium release from the sarcoplasmic reticulum and the subsequent increase in free cytosolic calcium levels is an essential component of excitation-contraction coupling in skeletal muscle (Ebashi et al. 1969). A number of recent studies have examined the metabolic consequences of caffeine-induced calcium release from the sarcoplasmic reticulum. These studies have shown that calcium induces an increase in glucose uptake (Wright et al. 2004, 2005, Jensen et al. 2007), an effect that could be blocked by inhibiting calcium release from the ryanodine receptor using dantrolene (Wright et al. 2004, 2005, Jensen et al. 2007), or by attenuating calcium/calmodulin kinase II (CaMKII) signalling (Wright et al. 2004, 2005, Jensen et al. 2007). Calcium release also stimulates fatty acid metabolism. For example, when cyclopiazonic acid, an inhibitor of sarcoplasmic/endoplasmic reticulum calcium ATPase (SERCA), was used to raise cytosolic calcium, fatty acid esterification was increased but fatty acid oxidation was not altered (Watt et al. 2003). In contrast, more recently, it was found that caffeine-induced calcium release stimulated fatty acid oxidation, an effect that could be blocked by inhibiting either CaMKII signalling (Raney \& Turcotte 2008), or calcium/calmodulin kinase kinase (CaMKK) signalling (Abbott et al. 2009). Clearly, caffeineinduced calcium release is sufficient to stimulate skeletal muscle fatty acid oxidation, but whether this stimulation is also dependent on caffeine-induced fatty acid transporter translocation to the sarcolemma has yet to be firmly established.

Fatty acid transport at the sarcolemma is regulated by a robust system of proteins. In addition to CD36, a number of other fatty acid transporters have been identified, including fatty acid-binding protein (FABPpm) and fatty acid transport protein (FATP) family members, FATP1 and FATP4. While these fatty acid transporters are known to reside on the sarcolemma and to facilitate fatty acid transport with different efficiencies (Nickerson et al. 2009), the responses of each transporter to various metabolic stimuli have only recently been examined. For instance, except for FATP6, all fatty acid transporters in muscle (FAT/CD36, FAB-
Ppm, FATP1 and 4) are induced to translocate by insulin and by muscle contraction (Jain et al. 2009). Nevertheless, several studies have suggested that metabolic stimulation of fatty acid oxidation in muscle is largely dependent on CD36 translocation to the sarcolemma (Bonen et al. 2007, Habets et al. 2007). In addition, caffeine-stimulated fatty acid oxidation has been linked with the translocation of CD36, but not FABPpm, to the plasma membrane (Abbott et al. 2009). However, in view of the presence of other fatty acid transporters in muscle, it is uncertain whether CD36 accounts primarily for the caffeine-induced changes in fatty acid oxidation. Moreover, it is unknown whether other fatty acid transporters (FATP 1 and 4) respond to caffeine stimulation or whether they can compensate for the loss of CD36 in KO mice.

In addition to being present on the sarcolemma, CD36 also resides within the mitochondrial compartment (Campbell et al. 2004) where it localizes to the outer mitochondrial membrane (Smith et al. 2011). In isolated mitochondria, contraction-mediated fatty acid oxidation is blunted in mitochondria isolated from CD36-null muscle (Holloway et al. 2009a), while in permeabilized muscle fibres, mitochondrial respiration, using palmitate as a substrate, is significantly reduced (Smith et al. 2011). It is therefore apparent that CD36 not only regulates fatty acid transport into muscle but it is also involved in regulating mitochondrial fatty acid metabolism. Whether the mitochondrial compartment contributes to calcium-induced increase in muscle fatty acid oxidation or whether this is CD36dependent is not known.

In this study, we use the CD36-null mouse to examine the effect of caffeine-induced calcium release on fatty acid transport and metabolism in skeletal muscle. Specifically, we sought to determine whether CD36 is solely responsible for caffeine-induced increases in fatty acid oxidation or whether other fatty acid transporters are also required. We also examined the contribution of mitochondrial CD36 to calcium-mediated fatty acid oxidation.

\section{Materials and methods}

\section{Animals}

Wild-type (WT) and CD36-null (KO) mice (gift, Dr. M. Febbraio, Cleveland Clinic, Cleveland, Ohio) were bred on site at the University of Guelph. Female mice (6-8 weeks of age) were housed in a temperature $\left(20{ }^{\circ} \mathrm{C}\right)$-regulated environment with a reversed 12 : 12-h light-dark cycle. Mice had access to standard laboratory chow and water ad libitum. The animal care committee at the University of Guelph approved all experimental procedures. 


\section{Fatty acid metabolism in isolated soleus muscles}

We examined the effects of caffeine on rates of fatty acid oxidation and esterification in WT-isolated soleus muscles. In addition, in WT and CD36-null soleus muscles, we determined the effects of caffeine and the combined effects of caffeine ( $3 \mathrm{~mm})$ and an inhibitor of calcium release (dantrolene, $10 \mu \mathrm{M}$ in DMSO; $0.3 \%$ DMSO final concentration) on fatty acid oxidation.

To determine palmitate esterification and oxidation, we used procedures that we have reported previously (Alkhateeb et al. 2007, Bonen et al. 2007). Briefly, soleus muscles were obtained from anesthetized mice (sodium pentobarbital, $6 \mathrm{mg} 100 \mathrm{~g}^{-1}$ body wt ip; MTC Pharmaceuticals, Cambridge, ON, Canada). To saturate the incubation media with oxygen, the incubation media (Media 199; Sigma-Aldrich, Oakville, ON, Canada) supplemented with 4\% BSA (Roche Diagnostics, Laval, QC, Canada) and $0.5 \mathrm{~mm}$ palmitate (Sigma) was pregassed for $40 \mathrm{~min}\left(95 \% \mathrm{O}_{2}\right.$ and $5 \% \mathrm{CO}_{2}$ ). To allow for recovery of the excised soleus muscles, they were preincubated in $2 \mathrm{~mL}$ of incubation media in a shaking water bath at $30{ }^{\circ} \mathrm{C}(100$ cycles per min) for $1 \mathrm{~h}$. Thereafter, muscles were transferred to new pregassed vials containing incubation media without (control) and with caffeine (3 mM), and unlabelled $(0.5 \mathrm{~mm})$ and $1-{ }^{14} \mathrm{C}$ palmitate $\left(0.5 \mu \mathrm{Ci} \mathrm{mL}{ }^{-1}\right) \quad$ (GE Healthcare Bio-Science, Baie d'Urfé, QC, Canada) and 4\% BSA. For inhibition studies, it was necessary to pre-expose muscles to $10 \mu \mathrm{M}$ dantrolene during the last $40 \mathrm{~min}$ of the preincubation period. Thereafter, muscles were transferred to incubation media containing radio-label as before, and $3 \mathrm{~mm}$ caffeine or $3 \mathrm{~mm}$ caffeine with $10 \mu \mathrm{M}$ dantrolene dissolved in DMSO $(0.3 \%$ final concentration). DMSO $(0.3 \%$ final concentration $)$ was also included in control muscles. In all experiments, mineral oil was layered on top of the media to prevent loss of radio-labelled $\mathrm{CO}_{2}$. The incubation was allowed to proceed for $60 \mathrm{~min}$. Thereafter, muscles were removed, blotted and were snap-frozen using a liquid $\mathrm{N}_{2}$ cooled clamp.

The procedures for palmitate oxidation and esterification have been used extensively by our group and have been reported previously in detail (Alkhateeb et al. 2007, Bonen et al. 2007). Briefly, ${ }^{14} \mathrm{CO}_{2}$ was released from the incubating media by the addition of sulphuric acid (1 M) and was captured (60 min) using a benzethonium hydroxide trap (Alkhateeb et al. 2007, Bonen et al. 2007). A Folch extraction was used to obtain lipid and aqueous fractions from homogenized muscle. Water-soluble ${ }^{14} \mathrm{C}$-labelled intermediate metabolites were measured in the aqueous phase, while ${ }^{14} \mathrm{C}$-palmitate incorporated into muscle triacylglycerol depots was measured in the organic phase using thin layer chromatography (Alkhateeb et al. 2007, Bonen et al. 2007).

\section{Oxygen consumption in isolated soleus muscle}

To determine whether oxygen consumption of WT and CD36-KO muscles was comparable, we isolated their soleus muscles as described above. The rate of oxygen consumption was determined using an Oxygraph 2-k (Oroboros Instruments, Innsbruk, Austria). Soleus muscles were added to the chambers containing pregassed incubation media (Media 199, supplemented with $4 \%$ BSA and $0.5 \mathrm{~mm}$ palmitate) at $30^{\circ} \mathrm{C}$ and stirring at $750 \mathrm{rpm}$. Once a basal steady state of $\mathrm{O}_{2}$ consumption had been achieved, $3 \mathrm{~mm}$ caffeine or media alone (basal) was added to the chamber. After these additions, the rate of $\mathrm{O}_{2}$ consumption was determined using Oroboros DatLab Software (Oroboros Instruments).

\section{Palmitate transport and plasma membrane fatty acid transporter content}

To obtain sufficient material for a single determination of palmitate transport in soleus muscles would have required the pooling of $30-50$ soleus muscles (6$8 \mathrm{mg}$ each). Therefore, we perfused murine hindlimb muscles and prepared giant sarcolemmal vesicles as we have described previously in detail (Bonen et al. 2007, Holloway et al. 2009a). Briefly, hindquarters were preperfused for $20 \mathrm{~min}$ after which the venous effluent was discarded. This was followed by $60-\mathrm{min}$ perfusion at $3 \mathrm{~mL} \mathrm{m^{-1 }}$ with a recirculating medium of $95 \% \mathrm{O}_{2} / 5 \% \mathrm{CO}_{2}$ Krebs-Henseleit buffer, $7 \mathrm{~mm}$ glucose, $0.5 \mathrm{~mm}$ palmitate and $4 \%$ bovine serum albumin, $\mathrm{pH} 7.4,37^{\circ} \mathrm{C}$. During the 60 -min experimental period, the hindlimb perfusions were performed without or with caffeine ( $3 \mathrm{~mm})$. The mouse hindlimb perfusion preparation is very stable, as we have established previously in our work (Bonen et al. 2007).

At the end of the perfusion period, giant sarcolemmal vesicles were generated as we have previously described (Bonen et al. 2000, Holloway et al. 2009a, Jain et al. 2009). Briefly, muscles from the perfused hindlimb were sectioned into 1 to 3 -mm-thick slices and were incubated for $1 \mathrm{~h}$ in $140 \mathrm{~mm} \mathrm{KCl}-10 \mathrm{~mm}$ MOPS [pH 7.4, collagenase type VII $\left(150 \mathrm{U} \mathrm{mL}^{-1}\right)$, aprotinin $\left(1 \mathrm{mg} \mathrm{mL}^{-1}\right)$ (Sigma-Aldrich)] while shaking in a $34^{\circ} \mathrm{C}$ water bath. When digestion was complete, muscle tissue was washed with $\mathrm{KCl}-\mathrm{MOPS}$ containing $10 \mathrm{~mm}$ EDTA. Percoll was added to the supernatant to give a final concentration of $3.5 \%$ Percoll, $28 \mathrm{~mm}$ $\mathrm{KCl}$ and $10 \mu \mathrm{g} \mathrm{mL}^{-1}$ aprotinin, and the resulting suspension was placed at the bottom of a density gradient consisting of a 1-mL KCl-MOPS upper layer and 
a middle layer of $4 \%$ Nycodenz (w/v). Following a $60 \mathrm{~g}$ centrifugation for $45 \mathrm{~min}$ at room temperature, the vesicles were harvested from the interface separating the upper and middle layers, diluted with $\mathrm{KCl}-$ MOPS and recentrifuged for $5 \mathrm{~min}$ at $12000 \mathrm{~g}$ at room temperature. For the determination of fatty acid transporter protein content, pellets were resuspended with $\mathrm{KCl}-\mathrm{MOPS}$ and stored at $-80^{\circ} \mathrm{C}$ for analysis by Western blotting.

Palmitate transport rates were measured as we have described previously in detail (Bonen et al. 2000, Holloway et al. 2009a, Jain et al. 2009). Briefly, $40 \mu \mathrm{L}$ of $0.1 \%$ BSA in $\mathrm{KCl}-\mathrm{MOPS}$, containing unlabelled $(15 \mu \mathrm{M})$ and radio-labelled $0.3 \mu \mathrm{Ci}\left[{ }^{3} \mathrm{H}\right]$-palmitate, and $0.06 \mu \mathrm{Ci}\left[{ }^{14} \mathrm{C}\right]$-mannitol, was added to $40 \mu \mathrm{L}$ of vesicle suspension. The incubation was carried out for 15 s. Palmitate uptake was terminated by the addition of $1.4 \mathrm{~mL}$ of ice-cold $\mathrm{KCl}-\mathrm{MOPS}, 2.5 \mathrm{~mm} \mathrm{HgCl}_{2}$ and $0.1 \%$ BSA. The sample was then centrifuged and the supernatant fraction was discarded. Thereafter, radioactivity was determined in the remaining pellet. Nonspecific uptake was measured by adding the stop solution before the addition of the radio-labelled palmitate solution.

\section{Palmitate oxidation by isolated mitochondria}

The effect of caffeine $(3 \mathrm{~mm})$ on mitochondrial fatty acid oxidation was determined in subsarcolemmal (SS) and intermyofibrillar (IMF) mitochondria isolated from perfused murine hindlimb muscles. For practical reason, it was not possible to do so in isolated murine soleus muscles, as this would have required pooling of 70-80 murine solei to obtain sufficient SS and IMF mitochondria for a single determination.

Hindlimb muscle perfusion was conducted as described above with or without caffeine (3 mM). After the perfusion period, SS and IMF mitochondria were isolated from pooled hindlimb muscles using differential centrifugation as we (Campbell et al. 2004, Holloway et al. 2007a) have previously described in detail. Briefly, muscles were removed and placed in Buffer 1 ( $50 \mathrm{~mm}$ Tris- $\mathrm{HCl}, 5 \mathrm{~mm} \mathrm{MgSO}_{4} \cdot 7 \mathrm{H}_{2} 0,5 \mathrm{~mm}$ EDTA, $100 \mathrm{~mm} \mathrm{KCl}$ ) at $4{ }^{\circ} \mathrm{C}$. They were trimmed of all tendons, weighed and then minced with scissors in $1 \mathrm{~mL}$ of Buffer 2 (Buffer 1 supplemented with $1.1 \mathrm{~mm}$ ATP). The muscle slurry was resuspended in $9 \mathrm{~mL}$ of Buffer 1 and homogenized for $12 \mathrm{~s}$ at $7500 \mathrm{rpm}$ using a polytron homogenizer. The homogenate was centrifuged at $800 \mathrm{~g}$, and the supernatant and pellet were saved. The supernatant was centrifuged at $9000 \mathrm{~g}$ to pellet the SS mitochondria. To liberate the IMF mitochondria, the pellet was resuspended in $4 \mathrm{~mL}$ of Buffer 2 and the muscle was digested with subtilisin A (Sigma) at a concentration of $0.25 \mu \mathrm{g} \mathrm{mg}^{-1}$ wet weight of muscle for exactly $5 \mathrm{~min}$ at $4{ }^{\circ} \mathrm{C}$. When digestion was complete, the suspension was centrifuged at $5000 \mathrm{~g}$, and the pellet was resuspended in $4 \mathrm{~mL}$ of Buffer 2. The resuspension was centrifuged at $800 \mathrm{~g}$, and the supernatant was retained and centrifuged at $9000 \mathrm{~g}$ to pellet IMF mitochondria. Freshly isolated SS and IMF mitochondria were used to determine palmitate oxidation in the presence of $\left[1-{ }^{14} \mathrm{C}\right]$ palmitate, as we have described previously (Campbell et al. 2004, Holloway et al. 2007a). Gaseous ${ }^{14} \mathrm{CO}_{2}$ production and isotopic fixation were determined in a comparable manner to that described for isolated soleus muscles (see above).

\section{Western blotting}

Protein analysis using soleus muscle homogenates was performed via Western blotting using standard procedures, as we have previously reported (Bonen et al. 2007, Jain et al. 2009). FAT/CD36 was detected using mouse monoclonal antibody CD36 (ME542) (Santa Cruz Biotechnology, Santa Cruz, CA, USA). FATP1 and FATP4 were detected using rabbit polyclonal IgG antibodies FATP1 (sc25541) and FATP4 (sc5834) (Santa Cruz). FABPpm was detected using antisera as we have reported previously (Holloway et al. 2009b, Jain et al. 2009). Signalling proteins of interest were detected using antibodies obtained from Cell Signaling Technology (Danvers, MA, USA), and the antibodies used were as follows: phospho-CaMKII (Thr286) Antibody \#3361, phospho-AMPK $\alpha$ (Thr172) Antibody \#2531, AMPK $\alpha$ antibody \#2532, phospho-Acetyl-CoA Carboxylase (Ser79) antibody \#3661 and Acetyl-CoA Carboxylase antibody \#3662. Immunodetection procedures were carried out as per the manufacturer's recommendations. Equal protein loading was confirmed by Ponceau staining. Protein images were obtained using enhanced chemiluminescent reagent (Western Lightning $^{\text {TM }}$ Plus-ECL; PerkinElmer, Waltham, MA, USA). Images were acquired using a Chemigenius2 system with GENESNAP software (Syngene, Cambridge, UK). Band densities were quantified using GeneTools software (Syngene).

\section{Statistics}

Data were analysed using two-way analysis of variance. When appropriate, a Fisher's LSD post hoc analysis was used. $P \leq 0.05$ was considered significant. All data are reported as mean \pm SEM.

\section{Results}

In WT soleus muscles, caffeine stimulated palmitate oxidation $(P<0.05$, Fig. 1$)$ while concurrently reduc- 
ing palmitate esterification $(P<0.05$, Fig. 1$)$. This net reduction in palmitate esterification could not account fully for the net increase in palmitate oxidation, as the increase in fatty acid oxidation $(\Delta=$ $\left.+40 \mathrm{nmol} \mathrm{g}{ }^{-1} 60 \mathrm{~min}^{-1}, P<0.05\right)$ was $54 \%$ greater than the reduction in fatty acid esterification $\left(\Delta=-26 \mathrm{nmol} \mathrm{g}^{-1} 60 \mathrm{~min}^{-1}\right)$. Therefore, subsequent experiments focused on fatty acid oxidation.

\section{Caffeine-induced increase in palmitate oxidation is blunted in CD36-KO mice}

To assess the role of CD36 in the caffeine-stimulated palmitate oxidation, soleus muscles from WT and CD36-KO mice were treated with (i) $3 \mathrm{~mm}$ caffeine or (ii) $3 \mathrm{~mm}$ caffeine plus dantrolene $(10 \mu \mathrm{M})$, an inhibitor of calcium release. Caffeine stimulated fatty acid oxidation in WT muscle $(+70 \%)$, but this caffeineinduced effect was 3.3-fold less in CD36-KO muscle $(+21 \%$ ) (Fig. 2a, $P<0.05)$.

Pretreatment of muscles with dantrolene inhibited caffeine-stimulated palmitate oxidation in both WT and CD36-KO muscles (Fig. 2b). However, in WT muscle, the dantrolene-mediated inhibition of palmitate oxidation was only partial $(-43 \%)$, while in CD36-KO muscles, this inhibition was complete (Fig. 2b).

A comparison of the net changes $(\Delta=$ caffeine - basal) in caffeine-stimulated fatty acid oxidation revealed a $70 \%$ lower response in the $\mathrm{KO}$ mice (Fig. 2c). This suggests that CD36 accounts for approx. $70 \%$ of caffeine-stimulated palmitate oxidation in soleus muscle.

We also measured basal and caffeine-stimulated oxygen consumption in isolated soleus muscle from

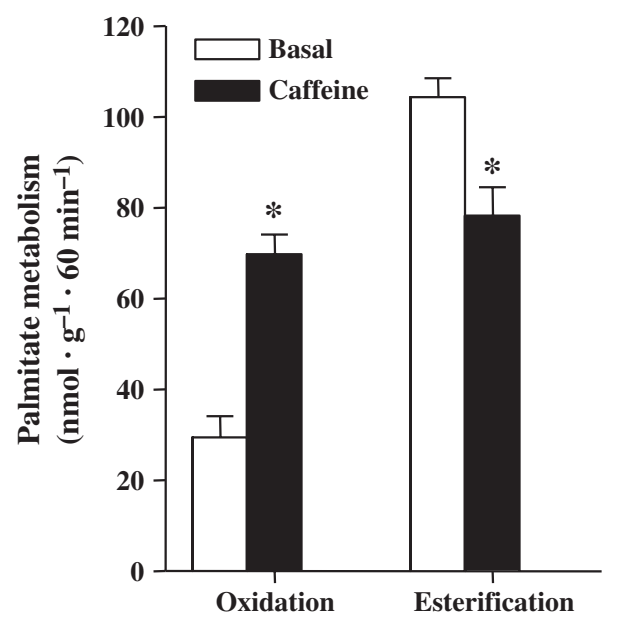

Figure I Caffeine ( $3 \mathrm{~mm}$ ) stimulated palmitate oxidation and esterification in WT mouse soleus muscle. $n=4-6$ muscles per treatment, data are presented as means \pm SEM; $* P<0.05$ caffeine vs. basal.
WT and CD36-KO mice (Fig. 2d). This is important because a decrease in the metabolic rate in $\mathrm{KO}$ muscle could account for their reduced rate of the palmitate oxidation. Under basal conditions, $\mathrm{O}_{2}$ consumption did not differ in WT and KO muscles $(P>0.05)$. Caffeine treatment increased $\mathrm{O}_{2}$ consumption in both WT $(+22 \%)$ and $\mathrm{KO}$ muscles $(+27 \% \mathrm{KO})(P<0.05)$, but there was no difference between WT and CD36-KO mice in the caffeine-stimulated rates of $\mathrm{O}_{2}$ consumption.

\section{Caffeine-induced fatty acid transport and fatty acid transporter translocation}

Owing to tissue limitations, it was not possible to determine fatty acid transport rates or sarcolemmal fatty acid transport protein content in isolated soleus muscle. Instead, palmitate transport rates and the plasma membrane content of fatty acid transporters CD36, FABPpm, FATP1 and FATP4 were determined in giant sarcolemmal vesicles prepared from caffeineperfused hindlimb muscles. As we have shown previously, basal fatty acid transport rates were reduced by $14 \%$ in $\mathrm{KO}$ muscle (Bonen et al. 2007). With caffeine stimulation, the fatty acid transport rate increased in both WT $(+55 \%)$ and KO (+16\%) muscles; however, as with palmitate oxidation, caffeine-induced fatty acid transport was severely inhibited in $\mathrm{KO}$ muscle (-70\%, Fig. 3a).

In WT muscle, increases in fatty acid transport rate were accompanied by concomitant increases in sarcolemmal content of all of the fatty acid transporters measured (CD36: $+37 \%$, FABPpm: $+26 \%$, FATP1: $+49 \%$ and FATP $4:+38 \%$, Fig. $3 \mathrm{~b}$ ). In KO muscle, as expected, CD36 was absent from the sarcolemma. There was no change in the basal sarcolemmal content of FATP1 protein; however, sarcolemmal FABPpm protein levels were reduced somewhat $(-25 \%)$ while sarcolemmal FATP4 content was increased $(+41 \%)$ in KO muscle. Despite these differences in basal sarcolemmal protein content in $\mathrm{KO}$ muscle, the caffeine-stimulated increases (\%) in FABPpm and FATP1 and 4 were comparable in CD36-KO and WT muscles (Fig. 3b).

\section{Mitochondrial fatty acid oxidation}

To ascertain whether the caffeine-induced increase in fatty acid oxidation at the whole muscle level was also associated with a stimulatory effect on mitochondria, we examined the effects of caffeine on fatty acid oxidation in isolated mitochondria. Under basal conditions, the rate of palmitate oxidation by IMF mitochondria was $80-90 \%$ greater than in SS mitochondria in WT and KO mice (Fig. 4). However, in 

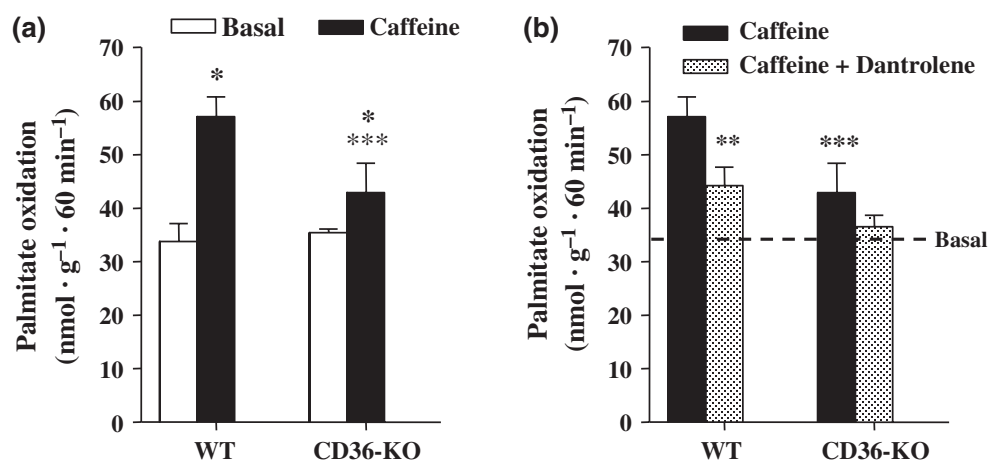

Figure 2 The effects of caffeine on palmitate oxidation and oxygen consumption in isolated soleus muscle of WT and CD36-KO mice. Panel (a): effects of caffeine ( $3 \mathrm{~mm}$ ) on palmitate oxidation, $n=8$. Panel (b): effects of caffeine $(3 \mathrm{~mm})+$ dantrolene $(10 \mu \mathrm{M})$ on palmitate oxidation, $n=8-9$. Dashed line represents the basal rate of palmitate oxidation shown in panel (a). Panel (c):
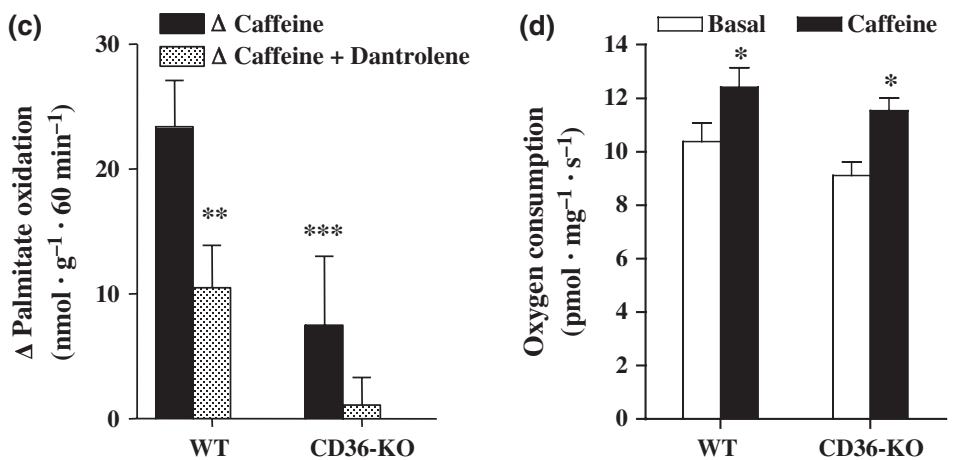

Net changes in palmitate oxidation: $\Delta=$ caffeine - stimulated fatty acid oxidation in individual muscle - average fatty acid oxidation in nonstimulated muscles. Panel (d): basal and caffeinestimulated oxygen consumption in isolated soleus muscle. $n=5-6$ muscles for each treatment, data are presented as means $\pm \mathrm{SEM} ; * P<0.05$, caffeine vs. control; $* * P<0.05$, WT caffeine + dantrolene vs. WT caffeine; $* * P<0.05$, KO caffeine vs. WT caffeine.

the $\mathrm{KO}$ animals, the basal rates of palmitate oxidation by isolated SS $(-23 \%)$ and IMF mitochondria $(-21 \%)$ were lower than in the WT mice. Perfusing muscle with caffeine failed to stimulate palmitate oxidation in either SS or IMF mitochondria either in WT or in $\mathrm{KO}$ mice (Fig. 4).

\section{Caffeine-induced phosphorylation of CaMKII, AMPK and ACC}

CaMKII, AMPK and ACC are important kinases in regulating the response to metabolic challenge in skeletal muscle and have been previously implicated in
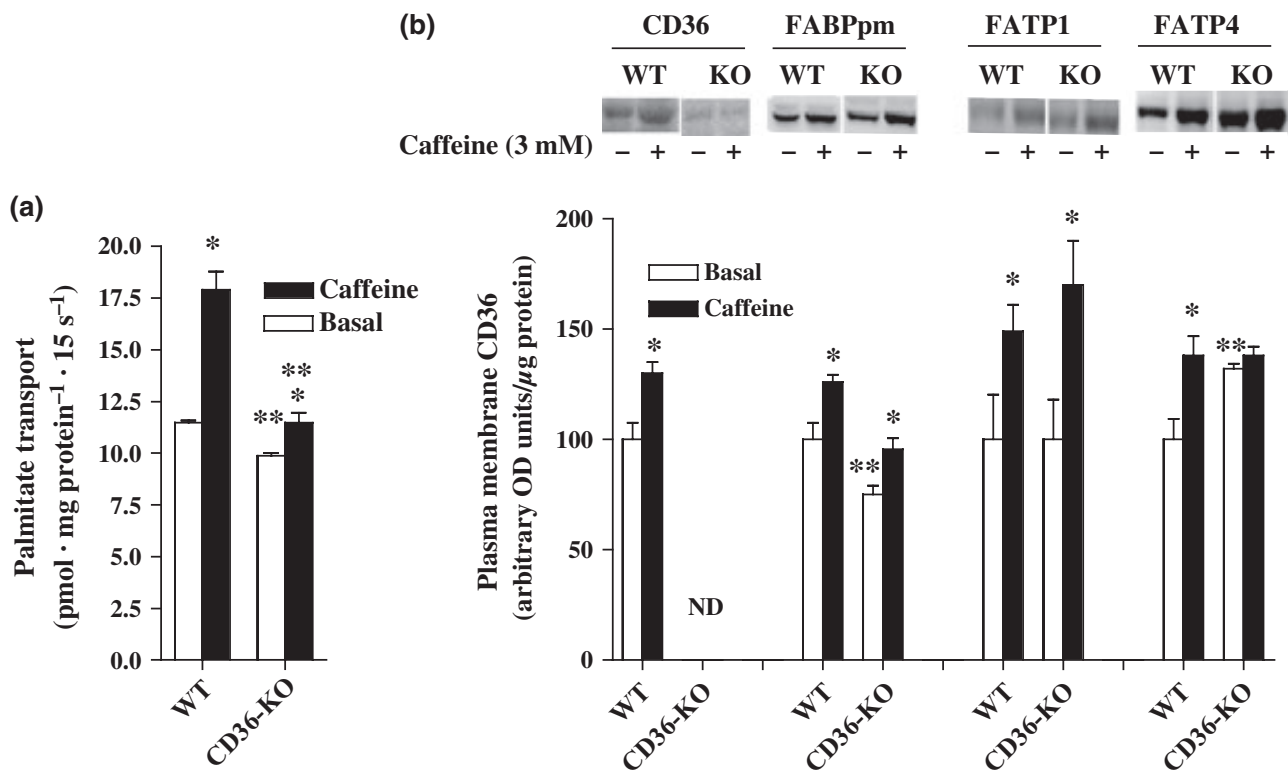

Figure 3 Caffeine stimulated fatty acid transport rates (a) and fatty acid transporter protein content (b) in giant sarcolemmal vesicles isolated from perfused hindlimb muscle of WT and CD36-KO mice. $n=4-5$ for each variable, data are presented as means $\pm \mathrm{SEM} ; * P<0.05$, caffeine vs. basal; $* * P<0.05$, KO significantly different from respective treatment in WT muscle; $\mathrm{ND}$, not detected. 


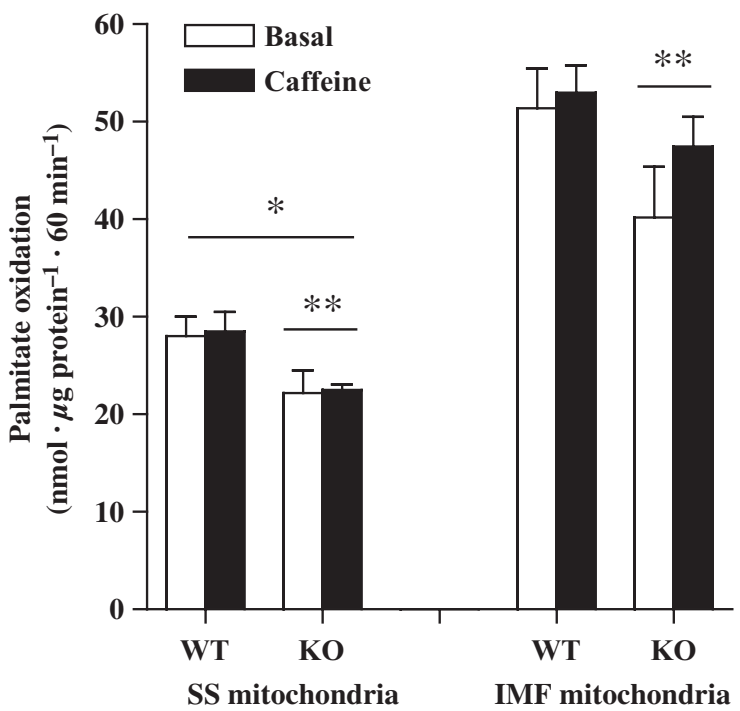

Figure 4 Fatty acid oxidation in isolated mitochondria from caffeine-stimulated hindlimb muscles from WT and CD36$\mathrm{KO}$ mice. For palmitate oxidation in isolated mitochondria, the muscles from each hindlimb were pooled for each determination. $n=5$ in each group, data are presented as means $\pm \mathrm{SEM} ; * P<0.05$, SS vs. IMF mitochondria in each of WT and KO mice; ${ }^{* * P}<0.05, \mathrm{KO}$ (basal and caffeine) vs. WT muscle (basal and caffeine). SS, subsarcolemmal; IMF, intermyofibrillar.

caffeine-stimulated fatty acid oxidation (Raney \& Turcotte 2008). Given the reduction in caffeine-stimulated palmitate oxidation in CD36-KO mice, we sought to determine whether the phosphorylation state of these kinases was also altered. As is common for the detection of signalling protein activations, the exposure to caffeine was necessarily brief (15 min) and therefore the protein expression of CaMKII, AMPK and ACC was not altered (data not shown).

CaMKII. Total CaMKII did not differ between WT and $\mathrm{KO}$ mice (data not shown). Basal CaMKII phosphorylation did not differ in WT and KO mice solei $(P>0.05)$. Exposure of soleus muscles to caffeine failed to induce CaMKII phosphorylation in either WT or KO mice $(P>0.05$, Fig. 5a).

AMPK. Total AMPK did not differ between WT and KO mice (data not shown). Basal AMPK phosphorylation did not differ in WT and KO mice solei $(P>0.05)$. Caffeine treatment of WT or KO solei increased AMPK phosphorylation comparably in both WT $(+36 \%)$ and CD36-KO mice $(+48 \%)(P<0.05$, Fig. 5b).

ACC. Total ACC did not differ between WT and KO mice (data not shown). Basal ACC phosphorylation did not differ in WT and KO mice solei $(P>0.05)$. This classical downstream target of AMPK was highly responsive to caffeine treatment, with ACC phosphorylation increasing in both WT and $\mathrm{KO}$ muscles $(P<0.05$, Fig. 5c). However, in WT soleus, caffeine increased ACC phosphorylation by $81 \%$, while in the CD36-KO muscles, ACC phosphorylation was increased significantly less $(+40 \%)(P<0.05$, Fig. 5c).

\section{Discussion}

We examined whether caffeine-stimulated fatty acid oxidation was dependent on CD36 translocation to the plasma membrane. We show that the ablation of CD36 completely abrogated the caffeine-induced upregulation of fatty acid oxidation, independent of changes in fatty (a)

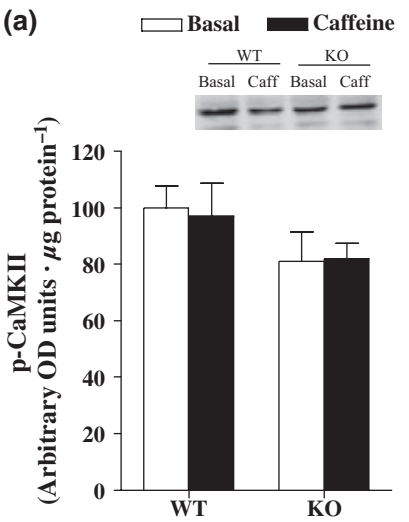

(b)

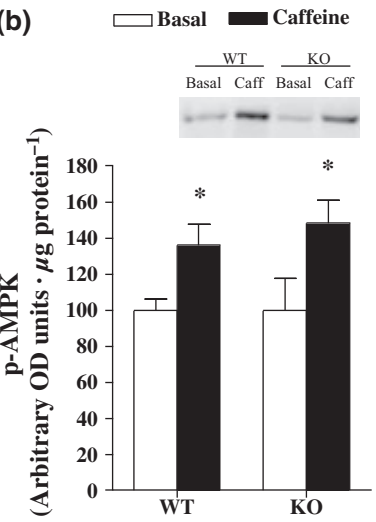

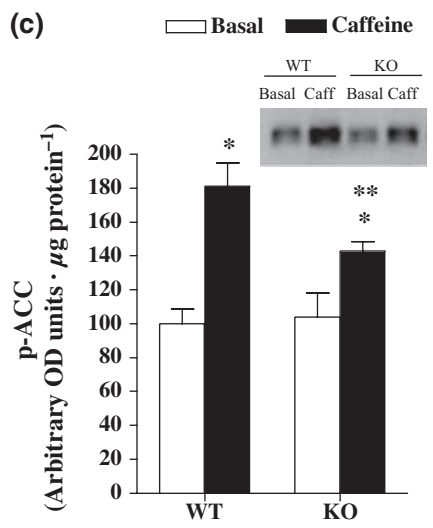

Figure 5 Caffeine stimulated CaMKII phosphorylation (Thr286) (a), AMPK $\alpha$ subunit phosphorylation (Thr172) (b), and ACC phosphorylation (Ser79) (c) in WT and CD36-KO mice. $n=5-6$ muscles for each treatment, data are presented as means \pm SEM. Equal quantities of protein were loaded for WT and CD36-KO samples and were routinely confirmed by Ponceau staining. The very brief stimulation with caffeine $(15 \mathrm{~min})$ does not alter the protein content of these proteins (data not shown). $* P<0.05$, caffeine vs. basal; ${ }^{* *} P<0.05$, KO caffeine vs. WT caffeine. 
acid oxidation by isolated SS and IMF mitochondria. Given the well-established role of CD36 in mediating fatty acid transport, we conclude that caffeine-stimulated fatty acid oxidation is regulated, in part, by the CD36mediated fatty acid transport across the sarcolemma.

\section{Caffeine induces CD36 translocation to plasma membrane}

Previous studies have established that CD36 is critically important in heart and skeletal muscle fatty acid metabolism (Glatz et al. 2010). CD36 is capable of binding long chain fatty acids (LCFA), and increases in plasma membrane CD36 are highly correlated with increases in fatty acid uptake into giant sarcolemmal vesicles (Glatz et al. 2010). In hearts and muscles of CD36 null mice, we have previously observed a greatly impaired rate of fatty acid uptake and oxidation when these tissues were stimulated with muscle contraction, AICAR or oligomycin (Bonen et al. 2007, Habets et al. 2007, Jain et al. 2009). Therefore, in the present study, the blunting of caffeine-stimulated fatty acid oxidation in CD36-null soleus muscle is likely due to the absence of CD36 on the plasma membrane. Supporting this notion are data in the present study and those of Abbott et al. (2009), which indicate that caffeine induces the translocation of CD36 to the plasma membrane.

In addition to CD36, translocation of other fatty acid transporters, namely FABPpm, FATP1 and FATP4, also occurred in response to caffeine stimulation. Yet, despite the responsiveness of other fatty acid transporters to caffeine, fatty acid transport and oxidation were reduced considerably in CD36 null mice. This is somewhat surprising, given the presence of other fatty acid transporters at the sarcolemma. However, there is some evidence that CD36 may be required for other transporters to achieve optimal efficiency. In particular, FABPpm and CD36 have been shown to coimmunoprecipitate with one another (Chabowski et al. 2007, S. Jain, and A. Bonen, unpublished data). While the association of CD36 with other fatty acid transporters is not yet firmly established, this could potentially explain the profound reduction in fatty acid uptake in the absence of CD36. Taken altogether, these data further illustrate the essential role of CD36 in skeletal muscle fatty acid metabolism at the sarcolemma.

\section{Mitochondrial fatty acid oxidation in response to caffeine}

Many laboratories (Campbell et al. 2004, Distler et al. 2006, Schenk \& Horowitz 2006, King et al. 2007, Aoi et al. 2008, Sebastian et al. 2009), except one (Jeppesen et al. 2010), have independently confirmed that CD36 is present in mitochondria, where it localizes to the outer mitochondrial membrane (Smith et al. 2011). Despite suggestions that these results reflected mitochondrial contamination (Jeppesen et al. 2010), many studies have shown that this is not the case (Bezaire et al. 2004, Campbell et al. 2004, Schenk \& Horowitz 2006, Holloway et al. 2007b, Smith et al. 2011). While the exact role of CD36 in mitochondria has not been fully established, we have recently shown, in CD36-KO mice, that this protein is required for muscle contraction-induced increases in fatty acid oxidation in isolated mitochondria (Holloway et al. 2009a) and that mitochondrial palmitate, but not palmitoyl-CoA, respiration is reduced in permeabilized muscle fibres of CD36-KO mice (Smith et al. 2011). Fatty acid oxidation can also be regulated by calcium at the level of the mitochondria, as $\mathrm{Ca}^{2+}$ is a well-known effecter on a number of mitochondrial enzymes. These include rate-limiting enzymes of the tricarboxylic acid cycle, namely isocitrate dehydrogenase (Denton et al. 1978) and $\alpha$-ketoglutarate dehydrogenase (McCormack \& Denton 1979). However, we did not observe any increase in palmitate oxidation in mitochondria isolated from caffeine-stimulated skeletal muscle in either CD36-KO or WT mice, indicating that mitochondrial CD36 is not necessary for the caffeine-induced increases observed at the whole muscle level. In this and other studies (Wright et al. 2004, Jensen et al. 2007, Raney \& Turcotte 2008, Abbott et al. 2009) the use of $3 \mathrm{~mm}$ caffeine is designed to release calcium into the cytosol without triggering muscle contraction (Youn et al. 1991). Given the lack of a calcium-induced increase in palmitate oxidation in isolated mitochondria, it appears that the calcium release elicited by $3 \mathrm{~mm}$ caffeine is perhaps too low to stimulate mitochondrial fatty acid oxidation, while still inducing the translocation of CD36 to the plasma membrane. It appears therefore that by stimulating CD36mediated fatty acid transport into muscle cells, the resultant increase in intracellular fatty acids is sufficient to stimulate fatty acid oxidation, without altering the intrinsic rate of palmitate oxidation by isolated mitochondria. This supports early work showing that increasing fatty acid provision to muscle stimulates their oxidation (Hickson et al. 1977). Our present findings further emphasize the key role of fatty acid transport, mediated by plasma membrane CD36, in the caffeineinduced upregulation of fatty acid oxidation. Indeed, the comparable increases in caffeine-stimulated $\mathrm{O}_{2}$ consumption $(+27 \%)$ and plasma membrane CD36 (+33\%) suggest that the translocation of this fatty acid transporter is tightly associated with muscle respiration rates.

\section{Caffeine-induced signalling}

Members of the CaMK family of kinases are activated by increases in cytosolic calcium/calmodulin (Corco- 
ran \& Means 2001) and are therefore candidates for the regulation of substrate utilization in response to calcium release, such as occurs during muscle contraction. CaMKII activation has been implicated in the translocation of both glucose and fatty acid transporters (Wright et al. 2004, Jensen et al. 2007, Raney \& Turcotte 2008, Witczak et al. 2010), but the precise role of CaMKII in this process remains elusive. Unfortunately, the majority of evidence in support of CaMKII activation by caffeine relies heavily on the use of the inhibitor KN-93 (Wright et al. 2004, Jensen et al. 2007, Raney \& Turcotte 2008), but recently the specificity of this inhibitor has been come into question (Witczak et al. 2010). In the present study, we have found that treatment with caffeine is not sufficient to cause CaMKII phosphorylation in mouse soleus muscle. Although there is evidence that muscle contraction can result in CaMKII phosphorylation in fast-twitch muscles (Witczak et al. 2010, Egawa et al. 2011), and in soleus muscle (A. Bonen, X-X. Han, and S. Jain, unpublished data), only a few studies have provided direct evidence that this kinase can be activated by caffeine treatment, and only in fast-twitch muscles (Wright et al. 2004). Studies examining caffeine effects on glucose transport in soleus muscle have not provided evidence that this treatment induces CaMKII phosphorylation in this slow-twitch muscle (Wright et al. 2005, Jensen et al. 2007). Taken altogether, it appears that caffeine-induced CaMKII phosphorylation may exhibit muscle fibre type specificity. Nonetheless, as CD36 translocation to the plasma membrane did occur, it appears that an increase in CaMKII phosphorylation may not be required for CD36 translocation in response to caffeine treatment.

A number of studies have implicated the AMPK signalling cascade in the regulation of glucose and fatty acid uptake in response to caffeine treatment (Jensen et al. 2007, Raney \& Turcotte 2008, Abbott et al. 2009, Egawa et al. 2011). We have also found that caffeine activates AMPK (as well as ACC), but there are a number of inconsistencies within the literature. Some have reported that caffeine fails to phosphorylate total AMPK in incubated soleus muscle (Wright et al. 2004), a measurement that correlates well with AMPK activity (Hawley et al. 2010). However, Jensen et al. (2007) showed that, while there was no change in total AMPK phosphorylation, caffeine treatment preferentially phosphorylated the AMPK alpha1 isoform, whereas alpha2 phosphorylation was unchanged. In epitrochlearis muscle, preferential activation of the alpha1 isoform occurred with $1 \mathrm{~mm}$ caffeine treatment, but the activity of both isoforms increased simultaneously with $3 \mathrm{~mm}$ caffeine treatment (Egawa et al. 2011). In contrast, others have shown that caffeine stimulation increases the activity of the alpha2 isoform and not alpha1 (Raney \& Turcotte 2008, Abbott et al. 2009). These conflicting results (Wright et al. 2004, Jensen et al. 2007, Raney \& Turcotte 2008, Abbott et al. 2009, Egawa et al. 2011) may be due to a number of factors, including differences in species (rat, Abbott et al. 2009, Raney \& Turcotte 2008, Egawa et al. 2011 vs. mouse, Jensen et al. 2007), experimental models (hindlimb perfusion, Abbott et al. 2009, Raney \& Turcotte 2008 vs. muscle incubation, Jensen et al. 2007, Egawa et al. 2011) or muscle fibre type distribution (fast-twitch, Abbott et al. 2009, Raney \& Turcotte 2008, Egawa et al. 2011 vs. slow-twitch muscles, Jensen et al. 2007). Although there is some discrepancy in the literature with respect to the caffeine-induced activation of AMPK, the observation that caffeine treatment increases ACC phosphorylation is consistent among studies (present study, Jensen et al. 2007, Raney \& Turcotte 2008, Abbott et al. 2009, Egawa et al. 2011).

Caffeine-stimulated phosphorylation of ACC was lower in muscle from KO mice than in WT mice. Phosphorylation decreases ACC activity (Carling et al. 1987), and the resultant decrease in malonyl-CoA levels relieves the inhibition on CPT- 1 and promotes fatty acid oxidation (McGarry et al. 1978). It is therefore possible that a reduction in caffeine-stimulated ACC phosphorylation in $\mathrm{KO}$ mouse muscle contributes to the overall reduction in fatty acid oxidation. However, recent studies using muscle-specific ACC2KO mice demonstrate that the deletion of ACC2 has no effect on palmitate oxidation (Olson et al. 2010), and whether AMPK is the exclusive kinase for ACC is equivocal (Dzamko et al. 2008). Given the importance of AMPK activation in the translocation of CD36 to the sarcolemma (Bonen et al. 2007, Habets et al. 2007, Jain et al. 2009), it appears that in response to caffeine-induced calcium release, AMPK activation serves to increase the uptake of fatty acids into muscle, thereby providing additional substrate to mitochondria and allowing an increase in fatty acid oxidation.

\section{Summary}

We have used a caffeine treatment to determine whether an increase in cytosolic calcium increases soleus muscle fatty acid oxidation in a CD36-dependent manner. We found that the caffeine-induced increases in (i) palmitate oxidation are blunted in CD36 null mice $(-70 \%)$, and that this is (ii) likely due to changes in the sarcolemmal content of CD36, but (iii) not to intrinsic changes in mitochondrial fatty acid oxidation. In addition, (iv) we show that the caffeine-stimulated increase in CD36 translocation, ACC 
phosphorylation and fatty acid oxidation occurs in concert with AMPK activation, which (v) is independent of CaMKII phosphorylation. Taken together, these observations support the critical role of plasma membrane CD36 in upregulating skeletal muscle fatty acid oxidation. Specifically, when muscle metabolism is increased by the release of calcium into the cytosol, CD36 is translocated to the plasma membrane, which allows for the uptake of additional fatty acids into muscle to provide more substrate to mitochondria to increase the rate of fatty acid oxidation.

\section{Conflict of interest}

The authors have no conflict of interest to disclose.

This work was funded by the Canadian Institutes of Health Research $(\mathrm{AB})$ and the Natural Sciences and Engineering Research Council of Canada (AB and GPH), and the European Community (Integrated Project LSHM-CT-2004-005272, Exgenesis) (JJFPL and JG). A. Bonen was the Canada Research Chair in Metabolism and Health.

\section{References}

Abbott, M., Edelman, A. \& Turcotte, L. 2009. CaMKK is an upstream signal of AMP-activated protein kinase in the regulation of substrate metabolism in contracting skeletal muscle. Am J Physiol Regul Integr Comp Physiol 297, 1724-1732.

Alkhateeb, H., Chabowski, A., Glatz, J.F., Luiken, J.F. \& Bonen, A. 2007. Two phases of palmitate-induced insulin resistance in skeletal muscle: impaired GLUT4 translocation is followed by a reduced GLUT4 intrinsic activity. Am J Physiol Endocrinol Metab 293, E783-E793.

Aoi, W., Naito, Y., Takanami, Y., Ishii, T., Kawai, Y., Akagiri, S., Kato, Y., Osawa, T. \& Yoshikawa, T. 2008. Astaxanthin improves muscle lipid metabolism in exercise via inhibitory effect of oxidative CPT I modification. Biochem Biophys Res Commun 366, 892-897.

Bezaire, V., Heigenhauser, G.J.F. \& Spriet, L.L. 2004. Regulation of CPT I activity in intermyofibrillar and subsarcolemmal mitochondria from human and rat skeletal muscle. Am J Physiol Endocrinol Metab 286, E85-E91.

Bonen, A., Luiken, J.J., Liu, S., Dyck, D.J., Kiens, B., Kristiansen, S., Turcotte, L.P., Van Der Vusse, G.J. \& Glatz, J. F. 1998. Palmitate transport and fatty acid transporters in red and white muscles. Am J Physiol 275, E471-E478.

Bonen, A., Luiken, J.J., Arumugam, Y., Glatz, J.F. \& Tandon, N.N. 2000. Acute regulation of fatty acid uptake involves the cellular redistribution of fatty acid translocase. J Biol Chem 275, 14501-14508.

Bonen, A., Han, X.-X., Habets, D.D.J., Febbraio, M., Glatz, J.F.C. \& Luiken, J.J.F.P. 2007. A null mutation in skeletal muscle FAT/CD36 reveals its essential role in insulin- and AICAR-stimulated fatty acid metabolism. Am J Physiol Endocrinol Metab 292, E1740-E1749.

Campbell, S.E., Tandon, N.N., Woldegiorgis, G., Luiken, J.J. F.P., Glatz, J.F.C. \& Bonen, A. 2004. A novel function for fatty acid translocase (FAT)/CD36: involvement in long chain fatty acid transfer into the mitochondria. $\mathrm{J}$ Biol Chem 279, 36235-36241.

Carling, D., Zammit, V.A. \& Hardie, D.G. 1987. A common bicyclic protein kinase cascade inactivates the regulatory enzymes of fatty acid and cholesterol biosynthesis. FEBS Lett 223, 217-222.

Chabowski, A., Górski, J., Luiken, J.J.F.P., Glatz, J.F.C. \& Bonen, A. 2007. Evidence for concerted action of FAT/ CD36 and FABPpm to increase fatty acid transport across the plasma membrane. Prostaglandins Leukot Essent Fatty Acids 77, 345-353.

Corcoran, E.E. \& Means, A.R. 2001. Defining Ca2+/calmodulin-dependent protein kinase cascades in transcriptional regulation. J Biol Chem 276, 2975-2978.

Denton, R.M., Richards, D.A. \& Chin, J.G. 1978. Calcium ions and the regulation of NAD+-linked isocitrate dehydrogenase from the mitochondria of rat heart and other tissues. Biochem J 176, 899-906.

Distler, A.M., Kerner, J., Peterman, S.M. \& Hoppel, C.L. 2006. A targeted proteomic approach for the analysis of rat liver mitochondrial outer membrane proteins with extensive sequence coverage. Anal Biochem 356, 18-29.

Dzamko, N., Schertzer, J.D., Ryall, J.G., Steel, R., Macaulay, S.L., Wee, S., Chen, Z.-P., Michell, B.J., Oakhill, J.S., Watt, M.J., Jørgensen, S.B., Lynch, G.S., Kemp, B.E. \& Steinberg, G.R. 2008. AMPK-independent pathways regulate skeletal muscle fatty acid oxidation. J Physiol (Lond) 586, 5819-5831.

Ebashi, S., Endo, M. \& Otsuki, I. 1969. Control of muscle contraction. Q Rev Biophys 2, 351-384.

Egawa, T., Hamada, T., Ma, X., Karaike, K., Kameda, N., Masuda, S., Iwanaka, N. \& Hayashi, T. 2011. Caffeine activates preferentially $\alpha 1$-isoform of 5'AMP-activated protein kinase in rat skeletal muscle. Acta Physiol (Oxf) 201, 227-238.

Febbraio, M., Abumrad, N.A., Hajjar, D.P., Sharma, K., Cheng, W., Pearce, S.F. \& Silverstein, R.L. 1999. A null mutation in murine CD36 reveals an important role in fatty acid and lipoprotein metabolism. J Biol Chem 274, 19055-19062.

Glatz, J.F., Luiken, J.J. \& Bonen, A. 2010. Membrane fatty acid transporters as regulators of lipid metabolism: implications for metabolic disease. Physiol Rev 90, 367-417.

Habets, D.D.J., Coumans, W.A., Voshol, P.J., Boer, M.A.M. D., Febbraio, M., Bonen, A., Glatz, J.F.C. \& Luiken, J.J.F. P. 2007. AMPK-mediated increase in myocardial longchain fatty acid uptake critically depends on sarcolemmal CD36. Biochem Biophys Res Commun 355, 204-210.

Hawley, S.A., Ross, F.A., Chevtzoff, C., Green, K.A., Evans, A., Fogarty, S., Towler, M.C., Brown, L.J., Ogunbayo, O. A., Evans, A.M. \& Hardie, D.G. 2010. Use of cells expressing gamma subunit variants to identify diverse mechanisms of AMPK activation. Cell Metab 11, 554-565.

Hickson, R.C., Rennie, M.J., Conlee, R.K., Winder, W.W. \& Holloszy, J.O. 1977. Effects of increased plasma fatty acids on glycogen utilization and endurance. J Appl Physiol 43, 829-833.

Holloway, G.P., Lally, J., Nickerson, J.G., Alkhateeb, H., Snook, L.A., Heigenhauser, G.J., Calles-Escandon, J., 
Glatz, J.F., Luiken, J.J., Spriet, L.L. \& Bonen, A. 2007a. Fatty acid binding protein facilitates sarcolemmal fatty acid transport but not mitochondrial oxidation in rat and human skeletal muscle. J Physiol 582, 393-405.

Holloway, G.P., Thrush, A.B., Heigenhauser, G.J.F., Tandon, N.N., Dyck, D.J., Bonen, A. \& Spriet, L.L. 2007b. Skeletal muscle mitochondrial FAT/CD36 content and palmitate oxidation are not decreased in obese women. Am J Physiol Endocrinol Metab 292, E1782-E1789.

Holloway, G., Jain, S., Bezaire, V., Han, X., Glatz, J., Luiken, J., Harper, M. \& Bonen, A. 2009a. FAT/CD36 null mice reveal that mitochondrial FAT/CD36 is required to up-regulate mitochondrial fatty acid oxidation in contracting muscle. Am J Physiol Regul Integr Comp Physiol 297, R960-R967.

Holloway, G.P., Benton, C.R., Mullen, K.L., Yoshida, Y., Snook, L.A., Han, X.-X., Glatz, J.F.C., Luiken, J.J.F.P., Lally, J., Dyck, D.J. \& Bonen, A. 2009b. In obese rat muscle transport of palmitate is increased and is channeled to triacylglycerol storage despite an increase in mitochondrial palmitate oxidation. Am J Physiol Endocrinol Metab 296, E738-E747.

Jain, S.S., Chabowski, A., Snook, L.A., Schwenk, R.W., Glatz, J.F.C., Luiken, J.J.F.P. \& Bonen, A. 2009. Additive effects of insulin and muscle contraction on fatty acid transport and fatty acid transporters, FAT/CD36, FABPpm, FATP1, 4 and 6. FEBS Lett 583, 2294-2300.

Jensen, T.E., Rose, A.J., Hellsten, Y., Wojtaszewski, J.O.F.P. \& Richter, E.A. 2007. Caffeine-induced $\mathrm{Ca}(2+)$ release increases AMPK-dependent glucose uptake in rodent soleus muscle. Am J Physiol Endocrinol Metab 293, E286-E292.

Jeppesen, J., Mogensen, M., Prats, C., Sahlin, K., Madsen, K. \& Kiens, B. 2010. FAT/CD36 is localized in sarcolemma and in vesicle-like structures in subsarcolemma regions but not in mitochondria. J Lipid Res 51, 1504-1512.

Kiens, B. 2006. Skeletal muscle lipid metabolism in exercise and insulin resistance. Physiol Rev 86, 205-243.

King, K.L., Stanley, W.C., Rosca, M., Kerner, J., Hoppel, C. L. \& Febbraio, M. 2007. Fatty acid oxidation in cardiac and skeletal muscle mitochondria is unaffected by deletion of CD36. Arch Biochem Biophys 467, 234-238.

Luiken, J.J.F.P., Dyck, D.J., Han, X.-X., Tandon, N.N., Arumugam, Y., Glatz, J.F.C. \& Bonen, A. 2002. Insulin induces the translocation of the fatty acid transporter FAT/ CD36 to the plasma membrane. Am J Physiol Endocrinol Metab 282, E491-E495.

McCormack, J.G. \& Denton, R.M. 1979. The effects of calcium ions and adenine nucleotides on the activity of pig heart 2-oxoglutarate dehydrogenase complex. Biochem J 180, 533-544.

McGarry, J.D., Takabayashi, Y. \& Foster, D.W. 1978. The role of malonyl-CoA in the coordination of fatty acid synthesis and oxidation in isolated rat hepatocytes. $J$ Biol Chem 253, 8294-8300.

Nickerson, J.G., Alkhateeb, H., Benton, C.R., Lally, J., Nickerson, J., Han, X.-X., Wilson, M.H., Jain, S.S., Snook, L.
A., Glatz, J.F.C., Chabowski, A., Luiken, J.J.F.P. \& Bonen, A. 2009. Greater transport efficiencies of the membrane fatty acid transporters FAT/CD36 and FATP4 compared with FABPpm and FATP1 and differential effects on fatty acid esterification and oxidation in rat skeletal muscle. J Biol Chem 284, 16522-16530.

Olson, D.P., Pulinilkunnil, T., Cline, G.W., Shulman, G.I. \& Lowell, B.B. 2010. Gene knockout of Acc2 has little effect on body weight, fat mass, or food intake. Proc Natl Acad Sci USA 107, 7598-7603.

Raney, M. \& Turcotte, L. 2008. Evidence for the involvement of CaMKII and AMPK in Ca2+-dependent signaling pathways regulating $\mathrm{FA}$ uptake and oxidation in contracting rodent muscle. J Appl Physiol 104, 13661373.

Schenk, S. \& Horowitz, J.F. 2006. Coimmunoprecipitation of FAT/CD36 and CPT I in skeletal muscle increases proportionally with fat oxidation after endurance exercise training. Am J Physiol Endocrinol Metab 291, E254E260.

Sebastian, D., Guitart, M., Garcia-Martinez, C., Mauvezin, C., Orellana-Gavalda, J., Serra, D., Gomez-Foix, A., Hegardt, F. \& Asins, G. 2009. Novel role of FATP1 in mitochondrial fatty acid oxidation in skeletal muscle cells. J Lipid Res 50, 1789-1799.

Smith, B.K., Jain, S.S., Rimbaud, S., Dam, A., Quadrilatero, J., Ventura-Clapier, R., Bonen, A. \& Holloway, G.P. 2011. FAT/CD36 is located on the outer mitochondrial membrane, upstream of long chain acyl-CoA synthetase, and regulates palmitate oxidation. Biochem J 437, 124134.

Watt, M.J., Steinberg, G.R., Heigenhauser, G.J.F., Spriet, L. L. \& Dyck, D.J. 2003. Hormone-sensitive lipase activity and triacylglycerol hydrolysis are decreased in rat soleus muscle by cyclopiazonic acid. Am J Physiol Endocrinol Metab 285, E412-E419.

Witczak, C.A., Jessen, N., Warro, D.M., Toyoda, T., Fujii, N., Anderson, M.E., Hirshman, M.F. \& Goodyear, L.J. 2010. CaMKII regulates contraction- but not insulininduced glucose uptake in mouse skeletal muscle. Am J Physiol Endocrinol Metab 298, E1150-E1160.

Wright, D.C., Hucker, K.A., Holloszy, J.O. \& Han, D.H. 2004. Ca2+ and AMPK both mediate stimulation of glucose transport by muscle contractions. Diabetes 53, 330-335.

Wright, D.C., Geiger, P.C., Holloszy, J.O. \& Han, D.-H. 2005. Contraction- and hypoxia-stimulated glucose transport is mediated by a $\mathrm{Ca} 2+$-dependent mechanism in slowtwitch rat soleus muscle. Am J Physiol Endocrinol Metab 288, E1062-E1066.

Youn, J.H., Gulve, E.A. \& Holloszy, J.O. 1991. Calcium stimulates glucose transport in skeletal muscle by a pathway independent of contraction. Am J Physiol 260, $555-561$. 